\title{
Bisantrene (NSC 337766) (CL 216,942) in advanced breast cancer
}

\section{A Cancer and Leukemia Group B study}

\author{
Arlene A. Forastiere', Michael C. Perry ${ }^{2}$, Ann K. Hughes ${ }^{3}$, and William C. Wood ${ }^{4}$ \\ ${ }^{1}$ University of Maryland Cancer Center, Division of Medical Oncology, 22 South Greene Street, Baltimore, MD 21201, USA \\ 2 University of Missouri Medical School, Division of Medical Oncology, 807 Stadium Blvd, Columbia, MO 65212, USA \\ ${ }^{3}$ Harvard University School of Public Health, Department of Biostatistics, 677 Huntington Avenue, Boston, MA 02115, USA \\ ${ }^{4}$ Massachusetts General Hospital, Department of Surgery, Blossom Street, Boston, MA 02114, USA
}

\begin{abstract}
Summary. Bisantrene (NSC 337766) is an anthracenedicarboxaldehyde hydrazone demonstrating $a$ wide spectrum of activity in animal tumor model systems with no evidence of cardiotoxicity or alopecia, in contrast to doxorubicin. Thirty-three women with advanced adenocarcinoma of the breast were treated with $260 \mathrm{mg} / \mathrm{m}^{2} \mathrm{IV}$ every 3 weeks. All patients had received at least one prior combination chemotherapy regimen for metastatic disease and 32/33 were refractory to doxorubicin. Of 28 patients evaluable for response one had a partial response lasting 10 weeks and three patients had stable disease for 22, 22, and 9 weeks. The most significant toxicities were nonhematologic: nausea and vomiting (41\%), phlebitic reactions (38\%), hypotension, one fatal anaphylactic reaction, and the development of a 7th cranial nerve palsy during drug infusion. Hematologic toxicity, leukopenia, was dose-limiting but manageable without associated infections or bleeding. These results indicate that bisantrene in this dose and schedule is not a useful drug in heavily pretreated breast cancer patients. The incidence and severity of phlebitic reactions limited venous access and adversely affected patient compliance. Preliminary results of other phase II breast cancer trials indicate a similar spectrum of toxicity but suggest more significant antitumor activity even in patients previously treated with doxorubicin. Trials conducted in patients with minimal prior treatment and with bisantrene administered via central line appear warranted for definitive assessment of the activity of this agent in breast cancer.
\end{abstract}

\section{Introduction}

Bisantrene (NSC 337766) (CL 216,942) (9,10-anthracenedicarboxaldehyde bis (4,5-dihydro-1H-imidazol-2-yl) hydrazone) dihydrochloride) is an anthracenedicarboxaldehyde hydrazone synthesized by American Cyanamid Co. in the interest of developing anthracene derivatives with a spectrum of activity similar to doxorubicin but without dose-limiting cardiotoxicity. In preclinical testing significant antitumor activity was observed in a wide spectrum of animal tumor models, including the mouse P-388 and L-1210 stem cell leukemias, Leiberman plasma cell tumor, B 16 melanoma,

Support in part by NCI Grants - CA 31983, CA 31946, CA 16118, CA 12449 , CA 33601

Offprint requests to: Arlene A. Forastiere (Present address: The University of Michigan Medical Center Simpson Memorial Institute, 102 Observatory, Ann Arbor, MI 48109, USA) colon tumor 26, and Ridgeway osteogenic sarcoma [4]. Although the exact mechanism of action is uncertain, bisantrene probably acts as a DNA-intercalating agent. In comparison with doxorubicin, bisantrene was shown to be three and four times more potent an inhibitor of ${ }^{3} \mathrm{H}$-uridine and ${ }^{3} \mathrm{H}$-thymidine incorporation into RNA and DNA, respectively, in the L5178Y lymphoma tissue culture system. The cytotoxic activity was not cell cycle-phase-specific [4]. Animal toxicology studies showed no evidence of alopecia or cardiomyopathy [4, $5,9]$. Significant antitumor activity against a number of tumors has been reported in the human tumor-cloning assay $[1,12]$. Results of breast cancer specimens tested for both bisantrene and doxorubicin sensitivity have shown bisantrene sensitivity in doxorubicin-resistant specimens, suggesting the absence of cross resistance between these two agents [8]. In phase I-II testing activity has been reported in renal cell carcinoma, breast carcinoma, and hepatocellular carcinoma $[1-3,6,8,11$, 13]. The dose-limiting toxicity was myelosuppression, predominantly leukopenia. Other side-effects included nausea and vomiting, phlebitis, hypotension, chills, urticarial reactions, and anaphylactoid reactions $[1,7,10]$. Based on this promising preliminary data the Cancer and Leukemia Group B initiated a phase II trial in women with advanced breast cancer.

\section{Materials and methods}

Thirty-three women with histologically confirmed adenocarcinoma of the breast were entered into the trial. Eligibility requirements included bidimensionally measurable disease, a performance score of 3 or less by CALGB criteria $(0=$ no impairment, $1=$ ambulatory, $2=$ in bed less than $50 \%$ of waking hours, $3=$ in bed more than $50 \%$ of waking hours, $4=$ bedridden), prior treatment with no more than two standard chemotherapy regimens and less than $550 \mathrm{mg} / \mathrm{m}^{2}$ doxorubicin, expected survival of at least 8 weeks, adequate bone marrow reserve: $\mathrm{WBC} \geqslant 4,000 / \mu \mathrm{l}$, platelets $>100,000 / \mu \mathrm{l}$, no evidence of obstructive liver disease: bilirubin $<1.5 \mathrm{mg} / \mathrm{dl}$, and normal renal function: serum creatinine $<1.5 \mathrm{mg} / \mathrm{dl}$. Before entering the study all patients had a complete history and physical examination, chest roentgenogram, complete blood count (CBC) with differential and platelet count, serum creatinine, electrolytes, serum liver function tests, 12-lead electrocardiogram and radionuclide left ventricular ejection fraction determination. Radionuclide liver and bone scans were obtained when clinically indicated. Patients with clinically evident cardiac disease or an ejection fraction less than 0.45 were ineligible. 
The initial dose for all patients was $260 \mathrm{mg} / \mathrm{m}^{2}$, diluted to a concentration of $0.1-0.5 \mathrm{mg} / \mathrm{ml}$ Dextrose in water (USP) infused over a period of no less than $2 \mathrm{~h}$. Treatment was repeated every 3 weeks. Dose modifications were based on the lowest count recorded during the preceding cycle as follows: if the $\mathrm{WBC}>3.000 / \mu \mathrm{l}$ and platelets $>100,000 / \mu \mathrm{l}$ bisantrene was increased to $280 \mathrm{mg} / \mathrm{m}^{2}$; with $\mathrm{WBC}<1,500 / \mu l$ or platelets $<$ $50,000 / \mu \mathrm{l}$ the dose was reduced to $220 \mathrm{mg} / \mathrm{m}^{2}$. Blood pressure was monitored at 15-min intervals during drug administration and for $60 \mathrm{~min}$ after completion of drug administration. CBC and platelet count were repeated weekly; serum liver function tests, serum creatinine, and measurable disease parameters were assessed every 3 weeks. An adequate trial of bisantrene required objective progression of disease after at least two cycles (6 weeks) or rapid progression after one cycle at a dose producing leukopenia (WBC $<3,000 / \mu 1$ ). Response criteria were defined as: partial response (PR), a reduction of at least $50 \%$ in the sum of the products of the greatest perpendicular diameters of all measurable disease for at least 4 weeks in the absence of new disease elsewhere; stable disease, objective regression of disease less than required for a PR or less than $25 \%$ increase in any measurable lesion; progression, increase in the sum of the products of the greatest perpendicular diameters of any measurable lesion by $25 \%$ or the appearance of new areas of malignant disease.

\section{Results}

Patient characteristics are detailed in Table 1. All patients had received at least one prior chemotherapy regimen for metastatic disease and two patients had, in addition, received adjuvant chemotherapy. In $63 \%$ of the patients there were two or more organ sites of disease.

Of the 33 patients entered on study, there were six inadequate trials, five due to drug toxicity. One patient experienced an anaphylactic reaction while receiving the second course of bisantrene and died of cardiogenic shock $12 \mathrm{~h}$ later. This patient had no prior history of cardiac disease. The first course of bisantrene had been well tolerated. Halfway through the infusion of the second course the patient became diaphoretic and hypotensive (palpable blood pressure of 60 $\mathrm{mm} \mathrm{Hg}$ ). The bisantrene infusion was discontinued and the patient's blood pressure responded to IV normal saline, corticosteroids, and epinephrine. However, due to the fluids given to elevate her blood pressure, the patient went into pulmonary edema and required intubation. Several hours after stabilization the patient again became hypotensive. Blood pressure could not be restored despite maximal efforts at cardiopulmonary resuscitation. One patient developed an irreversible 7 th cranial nerve palsy during infusion of the first dose of chemotherapy and was removed from study. Two patients with poor venous access developed edema of the arm after the first course of bisantrene infused via a peripheral vein. Both were taken off study by their physician although there was no clear progression of disease. One of these patients in. addition experienced hypotension during and after drug infusion. During the infusion the patient complained of dizziness and nausea. A change in blood pressure from 132/90 before treatment to $80 / 70 \mathrm{~mm} \mathrm{Hg}$ was recorded. The blood pressure responded to discontinuation of the infusion and the administration of hydrocortisone. The drug infusion was resumed and completed at a slower rate. However, $15 \mathrm{~min}$ after completion of the infusion the patient again became symptomatic and a blood pressure of $106 / 72$ was recorded. The patient received IV fluids and the blood pressure recorded 10 min later was $120 / 80$. No further problems occurred during an additional $1 \mathrm{~h}$ of observation. A fifth patient refused to continue treatment after experiencing severe nausea, vomiting, and 'numb' and 'cold' extremities after the first bisantrene

Table 1. Patient characteristics

\begin{tabular}{lc}
\hline No. evaluable for response & 28 \\
Median age (range) & $54(35-76)$ \\
Median performance score (range) & $1(0-3)$ \\
Prior chemotherapy & \\
Metastatic disease & $100 \%$ \\
Adjuvant & $8 \%$ \\
1 regimen & $42 \%$ \\
2 regimens & $58 \%$ \\
Average no. of cytotoxic drugs (range) & $5.2(3-7)$ \\
Dominant disease site & \\
Soft tissue & $48 \%$ \\
Osseous & $4 \%$ \\
Visceral & $48 \%$ \\
No. of courses of bisantrene & \\
1 & 9 \\
2 & 10 \\
3 & 3 \\
4 or more & 5 \\
Response & \\
Partial response & 1 \\
Stable & 3 \\
Progression & 24 \\
\hline
\end{tabular}

${ }^{a}$ Includes one early death from progressive disease

Table 2. Hematologic toxicity

\begin{tabular}{lc}
\hline & No. of patients \\
\hline No. of adequate trials & 27 \\
WBC (cells $/ \mu 1)$ & \\
$2,000-3,000$ & 11 \\
$1,000-1,900$ & 7 \\
$<1,000$ & 1 \\
Platelets (cells $/ \mu \mathrm{l})$ & \\
$50,000-100,000$ & 1 \\
$<50,000$ & 0 \\
Hb decrease $(\mathrm{g} \%)$ & \\
$2-4$ & 9 \\
\hline
\end{tabular}

${ }^{\text {a }}$ Lowest count recorded

Table 3. Nonhematologic toxicity

\begin{tabular}{lc}
\hline & No. of patients \\
\hline No. of evaluable patients & 32 \\
Nausea and vomiting & 13 \\
Diarrhea & 0 \\
Stomatitis & 2 \\
Phlebitis & 12 \\
Hypotension & 1 \\
Rash & 1 \\
Fever and chills & 2 \\
Neurotoxicity (cranial nerve palsy) & 1 \\
Anaphylactic reaction (fatal) & 1 \\
Alopecia & 0 \\
\hline
\end{tabular}


infusion. There was one early death from progressive disease prior to re-evaluation. This patient has been included as an evaluable failure to respond.

Of the 28 patients evaluable for response to bisantrene, 18 (65\%) received at least two cycles. There was one PR, occurring after three cycles in a patient with soft-tissue disease. This patient received eight cycles of bisantrene administered via Hickman catheter and had a response duration of 10 weeks. Three patients had stable disease for 22,22 , and 9 weeks. Two of these patients had Hickman catheters placed after five cycles and continued to receive treatment until there was objective progression of disease after six and seven cycles, respectively. The third patient, while demonstrating objective tumor regression, although less than that required for $\mathrm{PR}$, was removed from study after three cycles because of severe phlebitis. All other patients progressed.

Hematologic toxicity in the 27 patients in whom the trial of bisantrene was adequate is detailed in Table 2 . The dose was escalated in seven patients and reduced or delayed due to toxicity in four. Hematologic toxicity was manageable with no associated infections or bleeding episodes. Leukopenia (WBC $<3.000 / \mu \mathrm{l}$ ) was recorded in $70 \%$ of patients. Nonhematologic toxicity in 32 patients is detailed in Table 3 . Nausea and vomiting occurred in 13 (41\%) patients, grade 1 or 2 in nine (28\%), and grade 3 by CALGB criteria (more than two vomiting episodes per day) in four (13\%) patients. Only one patient received all drug infusions through a Hickman catheter. Phlebitic reactions occurred in 12 of the remaining 31 patients evaluable for nonhematologic toxicity. The onset of these reactions was observed up to $48 \mathrm{~h}$ after infusion and ranged from mild erythematous streaking along the vein to edema of the entire arm. Other significant toxicities have been detailed above.

\section{Discussion}

In this phase II trial of bisantrene for the treatment of advanced adenocarcinoma of the breast, only one major response was observed. This response result would preclude with $99 \%$ confidence a $15 \%$ response frequency with bisantrene in this dose and schedule. More favorable results have been reported in manuscript or abstract form by other investigators using a similar dose and schedule of bisantrene. Osborne et al. [8] reported six responses (two complete, four partial) in 30 patients, 19 of whom had received prior doxorubicin. Two of the responders were in the doxorubicin-pretreated group. Responders had received fewer prior drugs and had a better average performance status than nonresponders. The primary toxicity was leukopenia. An anaphylactoid reaction occurred in one patient; and five of six patients who received drugs via peripheral veins developed phlebitis. Yap et al. [13] have reported a $22 \%$ response rate, nine partial responses in 40 evaluable patients all previously treated with doxorubicin. However, of 23 patients known to be refractory to doxorubicin only two responded, as against seven responses in 14 patients who were not known to be refractory. Granulocytopenia was the most frequently encountered toxicity. Phlebitis occurred in all patients who received the drug via peripheral veins and one nonfatal acute anaphylactic reaction occurred. The Eastern Cooperative Oncology Group [3] observed two partial responses in 18 evaluable patients. Leukopenia, allergic reactions, and phlebitis were the dose-limiting toxicities. Cavalli et al. [2] have reported less encouraging preliminary results. One partial response was observed in 26 patients. These patients had received an average of six prior drugs and $50 \%$ of patients had been treated with doxorubicin. Leukopenia and phlebitis were noted as toxicities.

The patient population in this CALGB trial had been heavily pretreated, with $57 \%$ refractory to two prior combination chemotherapy regimens for metastatic disease and all but one patient previously treated with doxorubicin. The only major response occurred after three cycles of treatment. One other patient who was demonstrating tumor regression was taken off study after three cycles due to phlebitis. Although leukopenia was the dose-limiting toxicity as determined in phase I trials and the most frequently observed toxicity (70\%) in this trial, nonhematologic toxicities limited drug administration in six patients: phlebitis, nausea and vomiting, cranial nerve palsy, hypotension, and one fatal anaphylactic reaction. Difficulties with venous access and a patient population whose disease was resistant to many conventional agents, including doxorubicin, are possible reasons for the low activity observed in this trial. Preliminary results of other trials confirm a significant incidence of phlebitis when bisantrene is administered via peripheral veins despite dilution of bisantrene to concentrations less than $0.5 \mathrm{mg} / \mathrm{ml}$ and slow infusion rates. Hypotension and 'anaphylactoid' reactions have been reported in up to $10 \%$ of patients receiving multiple courses of bisantrene [7] and therefore represent a significant degree of morbidity and warrant cautious attention to drug administration. The preinfusion use of antihistamines and corticosteroids has been recommended, although benefit is uncertain since reactions have occurred in premedicated patients. However, bisantrene is well tolerated in most patients, producing only mild nausea and vomiting and no alopecia. Further trials of bisantrene, in patients with minimal prior treatment and with drug administered via central line, appear warranted to confirm its activity in breast cancer.

\section{References}

1. Alberts DS, Mackel C, Pocelinko R, Salmon SE (1982) Phase I clinical investigation of 9,10 -anthracenedicarboxaldehye-bis [(4,5-dihydro-1H-imidazol-2-yl)hydrozane] dichloride with correlative in vitro human tumor clonogenic assay. Cancer Res 42: 1170

2. Cavalli F, Claryse A, Bakkel Huinink WT, Clavel HM, Van Glabbeke M, Rozencweig M (1983) Phase II study of bisantrene in advanced breast cancer. Proc Am Soc Clin Oncol 2: C-441

3. Chang AY, Falkson G, Kaplan BH, Skeel RT, Muggia FM, Pandya KJ (1983) Phase II study of bisantrene $(9,10$ anthracene dicarboxaldehyde $\mathrm{Cl} 216,942$ ) in patients with refractory breast cancer. Proc Am Soc Clin Oncol 2: C-385

4. Citarella RV, Wallace RE, Murdock KC, Angier RB, Durr FE, Forbes M (1982) Activity of a novel anthracenyl bishydrazone, 9,10-anthracendicarboxaldehyde-bis [ 4,5 -dihydro-1H-imidazol2-yl]hydrazone) dichloride, against experimental tumors in mice. Cancer Res 42: 440

5. Medical Research Division, American Cyanamid Co. (1979) Investigators brochure: CL 216,942-9,10-anthracenedicarboxaldehyde bis-[(4,5-dibydro-1H-imidazol-2-yl)hydrazone] dihydrochloride. American Cyanamid, Pearl River

6. Myers JW, Von Hoff DD, Coltman CA Jr, Kuhn JG, Van Echo D, Rivkin S, Pocelinko R (1982) Phase II evaluation of bisantrene (CL 216,942) in advanced renal cell carcinoma. Cancer Treat Rep 66: 1869

7. Myers JW, Von Hoff DD, Kuhn JG, Osborne CK, Sandbach JF, Pocelinco R (1983) Anaphylactoid reactions associated with bisantrene infusions. Investigational New Drugs 1: 85 
8. Osborne CK, Von Hoff DD, Cowan JD, Sandbach J (1984) Bisantrene, an active drug in patients with advanced breast cancer. Cancer Treat Rep 68:357

9. Sparano BM, Gordon GH, Hall C, Iatropoulos MJ, Noble TF (1980) Assessment of cardiotoxic potential of a new anticancer compound, an anthracenebishydrazone derivative in beagle dogs: comparison with Adriamycin: In: Abstracts of the 20th Interscience Conference on Antimicrobial Agents and Chemotherapy, Abstracht \#31

10. Spiegel RJ, Blum RH, Levin M, Pinto CA, Wernz JC, Speyer JL, Hoffman KS, Muggia FM (1982) Phase I clinical trial of 9,10-anthracenedicarboxaldehyde (bisantrene) administered in a five-day schedule. Cancer Res 42:354

11. Von Hoff DD, Myers JW, Kuhn J, Sandbach JF, Pocelinko R,
Clark G, Coltman CA (1981a) Phase I clinical investigation of 9-10 anthracenedicarboxaldehyde-bis[(4,5-dihydro- $1 \mathrm{H}$-imidazol-2-yl) hydrazone] dihydrochloride (CL 216,942). Cancer Res $41: 3118$

12. Von Hoff DD, Coltman CA Jr, Forseth B (1981b) Activity of 9,10-anthracenedicarboxaldehye-bis[(4,5-dihydro-1H-imidazol2-yl)hydrazone] dichloride (CL 216,942) in a human tumor cloning system. Leads for phase II trials in man. Cancer Chemother Pharmacol 6:141

13. Yap H, Yap B, Blumenschein GR, Barnes BC, Schell FC, Bodey GP (1983) Bisantrene, an active new drug in the treatment of metastatic breast cancer. Cancer Res 43:1402

Received December 12, 1983/Accepted April 13, 1984 